\title{
Salidroside Attenuates Ventilation Induced Lung Injury via SIRT1-Dependent Inhibition of NLRP3 Inflammasome
}

\author{
Yan Wang ${ }^{a, c}$ Chu-Fan Xua,c Yu-Jian Liư Yan-Fei Mao ${ }^{a}$ Zhou Lva Si-Yuan Lia \\ Xiao-Yan Zhu ${ }^{b}$ Lai Jiang ${ }^{\text {a }}$ \\ aDepartment of Anesthesiology and Surgical Intensive Care Unit, Xinhua Hospital, Shanghai \\ Jiaotong University School of Medicine, ${ }^{b}$ Department of Physiology and The Key Laboratory of \\ Molecular Neurobiology of Ministry of Education, second military medical university, 'School of \\ Kinesiology, The key Laboratory of Exercise and Health Sciences of Ministry of Education, Shanghai \\ University of Sport, Shanghai, China
}

\section{Key Words}

Salidroside • Ventilation-induced lung injury $•$ NLRP3 inflammasome • SIRT1 • Cyclic stretch

\begin{abstract}
Background: Salidroside (SDS) is the main effective ingredient of Rhodiola rosea $L$ with a variety of pharmacologic properties. We aim to investigate the effects of SDS on ventilation induced lung injury (VILI) and explore the possible underlying molecular mechanism. Methods: Lung injury was induced in male ICR mice via mechanical ventilation $(30 \mathrm{ml} / \mathrm{kg})$ for 4h. The mice were divided in four groups:(1) Control group; (2) Ventilation group; (3) SDS group; (4) Ventilation with SDS group. SDS (50 mg/kg) was injected intraperitoneally $1 \mathrm{~h}$ before operation. Mouse lung vascular endothelial cells (MLVECs) were subjected to cyclic stretch for 4h. Results: It was found that SDS attenuated VILI as shown in HE staining, cell count and protein content levels in BAL fluid, W/D and Evans blue dye leakage into the lung tissue. SDS treatment inhibited the activation of NLRP3 inflammasome and subsequent caspase- 1 cleavage as well as interleukin (IL)- $1 \beta$ secretion both in vivo and in vitro. Moreover, SDS administration up-regulated SIRT1 expression. Importantly, knockdown of SIRT1 reversed the inhibitory effect of SDS on NLRP3 inflammasome activation. Conclusions: Taken together, these findings indicate that SDS may confer protection against ventilation induced lung injury via SIRT1-dependent inhibition of NLRP3 inflammasome activation.

\section{Introduction}

Mechanical ventilation is widely used in the intensive care unit for patients with acute respiratory distress syndrome (ARDS). Whereas, ventilation is increasingly recognized

Y. Wang and C.-F. Xu contributed equally to this work and should be considered as co-first authors

Dr. Lai Jiang, or Dr. Xiaoyan Zhu

KARGER
Department of Anesthesiology and Surgical Intensive Care Unit, Xinhua Hospital, Shanghai Jiaotong University School of Medicine, Shanghai 200092, (China) or Department of Physiology, Second Military Medical University, 800 Xiangyin Road, Shanghai 200433 (China); E-Mail jianglaimz@126.com / xiaoyanzhu@smmu.edu.cn 
for its detrimental effects on pulmonary integrity and can trigger ventilator-induced lung injury(VILI) [1].The etiology of VILI is most likely multifactorial and it is characterized by an increasing level of lung proinflammatory mediators and increased vascular permeability, which leads to pulmonary edema [2,3]. As an undesirable side reaction of the routinely used therapy, effective treatments for treating VILI need to be more clearly identified.

The NOD-like receptor family, pyrin domain-containing 3 (NLRP3) inflammasome is a multiprotein cytoplasmic complex that comprised of NLRP3, caspase-1 and apoptosisassociated speck-like protein (ASC), leading to maturation of the proinflammatory cytokines interleukin (IL)-1 $\beta$ and IL-18 [4]. Previous studies have shown that the aberrant activation of NLRP3 inflammasome has been shown to contribute to mechanical ventilator-induced lung inflammation and injury [5]. The NLRP3 inflammasome is a pivotal signaling platform that is activated by a variety of signals, such as reactive oxygen species (ROS) [6]. Activation of NLRP3 inflammasome can release cytokines, growth factors, extracellular matrix (ECM) proteins and metalloproteases, thus causing alveolar injury, abnormal lung repair and pulmonary fibrosis [7]. As an innate proteolytic complex, the activation of NLRP3 inflammasome is recently known to play a critical role in the pathogenesis of ventilator-induced lung injury, thus the activation of NLRP3 inflammasome should be efficiently controlled.

Sirtuin 1 (SIRT1), a class III histone deacetylase, plays a pivotal role in variety of cellular processes ranging from gene silencing, control of the cell cycle and apoptosis, to metabolism and aging [8]. A recently report proves that SIRT1 inhibits the activation of NLRP3 inflammasome in mesenchymal stem cells treated with radiation [9]. In addition, Li et al. also demonstrate that SIRT1 exerts potential anti-inflammatory effect through regulation of the NLRP3 inflammasome in vascular endothelial cells (ECs) [10]. These findings suggest a direct inhibitory effect of SIRT1 on the activation of NLRP3 inflammasome.

Salidroside (SDS) is an active ingredient extracted from Rhodiola rosea, which has been used as a medicine herb to relieve high altitude sickness and improve resistance to fatigue [11]. Nowadays, salidroside receives increasing attention for its various pharmacological functions, among which include anti-inflammation, antioxidation, antitumor, and neuroprotection [12-14]. It has demonstrated that SDS can mitigate acute lung injuries in lipopolysaccharide- or paraquat-challenged animals $[15,16]$. Although the antiinflammatory effect of salidroside is emerging, the mechanism and the application in VILI are still poorly understood.

Therefore, in the present study, we first investigated the potential role of salidroside on NLRP3 inflammasome activation in VILI. Then, we researched whether SIRT1 was involved in the inhibition effect of salidroside against NLRP3 inflammasome induction so as to elucidate its anti-inflammatory mechanisms.

\section{Materials and Methods}

Animal preparation and salidroside treatment

All experimental protocols were approved by Shanghai Jiaotong University School of Medicine and the methods were carried out in accordance with the approved guidelines. Male ICR (25-30 g body weight) mice were purchased from the Animal Experimentation Center of Second Military Medical University and handled on a 12-h light/dark cycle at a constant temperature with unlimited access to food and water. Ventilation-induced lung injury was performed as described previously [17]. In short, mice were anesthetized with ketamine $(70 \mathrm{ml} / \mathrm{kg}$ ) and xylazine $(10 \mathrm{mg} / \mathrm{kg}$ ) and connected to a ventilator (Inspira, Harvard Apparatus Ltd, Boston, MA, USA) via establishing a tracheotomy ( $30 \mathrm{ml} / \mathrm{kg}$ at 70 breaths $/ \mathrm{min}$ ) for 4 hours. The mice were randomly assigned to four groups (7 mice per group): control group(Con), ventilation group(Ven), salidroside group(SDS), ventilation plus salidroside group(Ven+SDS). Salidroside (50 mg/kg, Sigma-Aldrich, St Louis, MO, USA) was injected intraperitoneally an hour before ventilation. Mice in Shamoperated groups were administrated with an equal volume of $0.9 \%$ saline. After ventilation, all animals were sacrificed and collected bronchoalveolar lavage fluids (BAL) or the lung tissues were removed then fixed in $4 \%$ paraformaldehyde or used for lung wet-to-dry ratio (W/D) or stored in $-80^{\circ} \mathrm{C}$ before assay. 


\section{Cellular Physiology Cell Physiol Biochem 2017;42:34-43

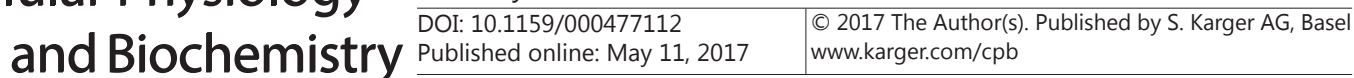

Wang et al.: SDS Attenuates VILI

Histological examination

The fixed lung tissues were embedded in paraffin, cut into 4- $\mu \mathrm{m}$-thick slices, and incubated with hematoxylin and eosin (H\&E) for histological examination. The degree of lung inflammation in the alveolar tissue were assessed by two blinded pathologists with expertise in lung pathology and graded as previous reported [17]: $0=$ normal tissue; $1=$ tiny inflammatory change; $2=$ mild to moderate inflammatory changes (no marked damage to the lung architecture); $3=$ moderate inflammatory injury (thickening of the alveolar septa); $4=$ =moderate to severe inflammatory injury(formation of nodules or areas of pneumonitis); and $5=$ severe inflammatory injury (total obliteration of the field).The mean score was reported per section.

\section{Western blotting analysis}

Lung tissues (20 mg) or MLVECs were homogenized and lysed with cold RIPA (Beyotime, China) containing 1\% proteinase Inhibitor Cocktail (Sigma). Then the lysates were centrifuged at $12000 \mathrm{rpm}$ for $20 \mathrm{~min}$ at $4{ }^{\circ} \mathrm{C}$ and the supernatant was collected. Equal amounts of proteins $(40 \mu \mathrm{g})$ were separated with SDS-PAGE, transferred to PVDF membrane (Millipore Corp, Bedford, MA, USA), blocked with 5\% BSA, and incubated with primary antibodies against NLRP3(Cell Signaling Technology, Beverley, CA), SIRT1(CST), caspase-1(Santa Cruz Biotechnology, Santa Cruz, CA), $\beta$-actin (Santa Cruz) respectively. Detection of immunoreactive bands were performed using BeyoECL Plus reagents (Millipore). The expression of $\beta$-actin was used as an internal control.

Real-time RT-PCR

The primers of NLRP3(NM_145827.3), ASC(NM_023258.4) and $\beta$-actin(NM_007393.3) for quantitative real-time PCR were as follows:

NLRP3 Forward,5'-CCACAGTGTAACTTGCAGAAGC-3';

NLRP3 Reverse,5'-GGTGTGTGAAGTTCTGGTTGG-3';

ASC Forward,5'-AAAGAAGAGTCTGGAGCTGTGG-3';

ASC Reverse,5'-GCAATGAGTGCTTGCCTGT-3';

$\beta$-actin Forward,5'-CTGTATGCCTCTGGTCGTAC-3';

$\beta$-actinReverse, 5'-TGATGTCACGCACGATTTCC-3';

Total RNAs from lung tissue were isolated and then processed into cDNA. Real-time PCR was carried out using SYBR green (F.Hoffmann-La Roche Ltd, Basel, Switzerland). Quantitative real-time PCR was carried out using StepOne Plus (Applied Biosystem, FosterCity, CA) apparatus. The method $2^{-\Delta \Delta \mathrm{Ct}}$ was used to determine the relative quantitation of target gene expression. Endogenous $C t$ values of $\beta$-actin were used as a control.

\section{RNA Interference}

SIRT1 small interfering RNA (siRNA) was synthesized by Genepharma Corp. (Shanghai, China). The sequences of mouse SIRT1 siRNA were: 5'-UUG CAGAAACAGUGAGAAA-3'. The negative control siRNA was from Genepharma. Transfection of siRNA was performed by using the Xfect siRNA transfection reagents (Takara, Japan).

\section{Cell culture and treatment}

MLVECs were isolated as previously described [17] and cultured at $37^{\circ} \mathrm{C}$ and $5 \% \mathrm{CO} 2$ in Dulbecco's modified Eagle's medium (DMEM; HyClone, Logan, UT, USA) added with $10 \%$ fetal bovine serum (FBS; Gibco, Grand Island, NY, USA). For cyclic stretch, MLVECs were seeded onto Collagen I coated Bioflex ${ }^{\circledR}$ six- $^{-}$ well culture plates. After $24 \mathrm{~h}$ of transfection of SIRT1 siRNA, the medium was changed and the cells were exposed to high-magnitude ( $20 \%$ linear elongation, sinusoidal wave, $30 \mathrm{cycles} / \mathrm{min}$ ) for 4 hours, by using the Flexcell@ FX-5000 Tension System. Control Bioflex plates with static cells were placed in the same incubator next to cyclic stretched cells. After mechanical stretch, the cells and culture supernatants were collected for analyses.

\section{Statistical analysis}

All data were expressed as means \pm SEM. The comparison between more than 3 groups was estimated by one-way analysis of variance followed by LSD test. A level of $p<0.05$ was considered statistically significant. SPSS version 16.0.1 was used to complete all the calculations. 


\section{Results}

\section{Effects of salidroside on ventilation induced acute lung injury}

To investigate the effects of salidroside on VILI, mice were exposed to high tidal volume ventilation and treated with salidroside (50 mg/kg) for 4 hours. As shown in Fig.1A and 1B, mechanical ventilation exhibited significant lung injury as indicated by prominent increases in cell counts and protein content levels in BAL fluid, which were inhibited by salidroside treatment. Salidroside alone had no effect on the cell count or protein content levels in BAL fluid.

Then the role of salidroside in the development of VILI was further assessed by histopathological examination, lung W/D and measurement of Evans blue leakage into the lung tissue (Fig. 2 and Fig. 1C-1D). The lung injury score was significantly increased after ventilation challenge compared with that in the control group, whereas was markedly reduced by the administration of salidroside. Meanwhile, mechanical ventilation induced edema and Evans blue leakage from the vascular space into the lung parenchyma in mice, which were alleviated obviously in the mice treated with salidroside. Salidroside treatment alone had no significant effect on histological changes, lung W/D and Evans blue tissue accumulation in the lungs.

Collectively, our findings strongly indicated that salidroside exerted protective effects against the development of VILI.

Salidroside inhibits ventilation-induced activation of NLRP3 inflammasome in lung tissues Kuipers et al. have demonstrated the activation of NLRP3 inflammasome is involved in the mechanical ventilator-induced acute lung injury [5]. We then evaluated the role of Salidroside in ventilation-induced activation of the NLRP3 inflammasome. Consistent with previous studies, we showed obviously increases in pulmonary NLRP3 and ASC expressions (Fig. 3A and 3B), as well as levels of cleaved caspase-1, IL-1 $\beta$ and IL-18 (Fig. 3C-E) in the ventilation group compared with the control group. Salidroside treatment significantly attenuated mechanical ventilation-induced increases in NLRP3, ASC, cleaved caspase-1, IL-1 $\beta$ and IL-18 in the lung tissues (Fig. 3). Taken together, these results indicated that Salidroside played a vital role in inhibiting ventilation-induced activation of NLRP3 inflammasome in vivo.

Fig. 1. Effects of salidroside on ventilation induced acute lung injury. Mice were subjected to mechanical ventilation $(30 \mathrm{ml} / \mathrm{kg}$ ) for 4h. Salidroside was intraperitoneally administered $1 \mathrm{~h}$ before the onset of ventilation. Cell count(A) and measurements of protein concentration(B) were performed in BAL fluid. Lung W/D(C) was measured as an index of pulmonary edema. Pulmonary vascular leak(D) was analyzed by Evans blue-labeled albumin extravasation into the lung tissue. Data are expressed as the mean \pm SEM $(\mathrm{n}=7) .{ }^{* *} \mathrm{p}<0.01$ vs control group; $\# \# p<0.01$ vs ventilation group.
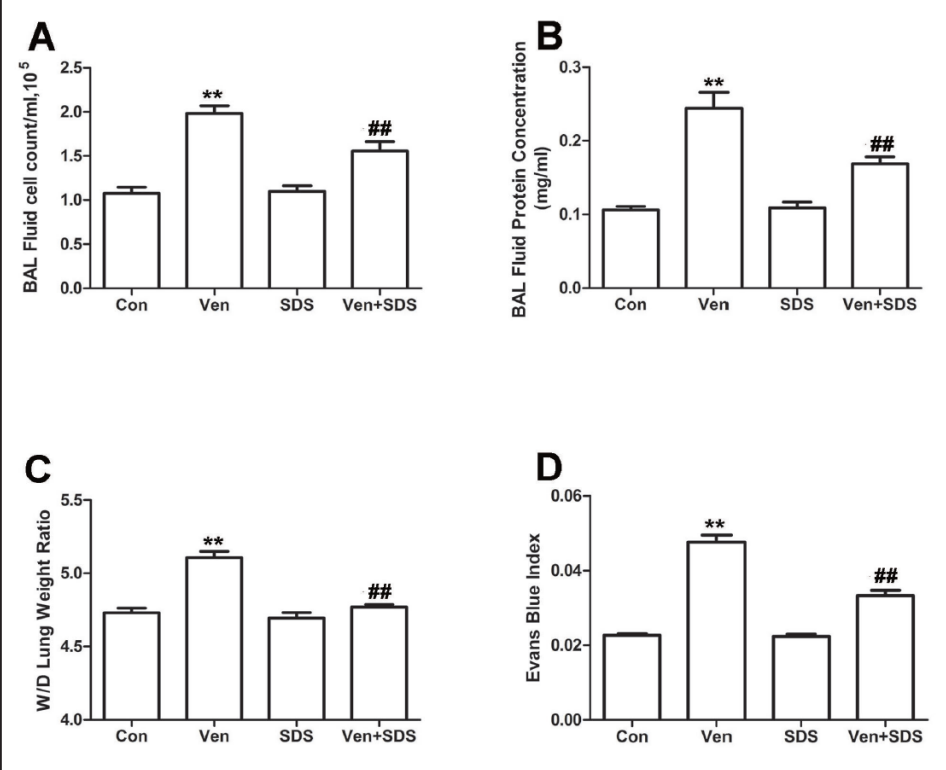


\begin{tabular}{|c|c|c|}
\hline \multirow{2}{*}{$\begin{array}{l}\text { Cellular Physiology } \\
\text { and Biochemistry }\end{array}$} & \multicolumn{2}{|c|}{ Cell Physiol Biochem 2017;42:34-43 } \\
\hline & $\begin{array}{l}\text { Dol: } 10.1159 / 000477112 \\
\text { Publisned online: } 2 \text { May II. } 2017\end{array}$ & $\begin{array}{l}\text { O } 2017 \text { The Author(s). Published by S. Karger AG, Basel } \\
\text { www.karger.com/cpb }\end{array}$ \\
\hline
\end{tabular}

Fig. 2. Salidroside treatment improves lung histopathological changes in the lung tissues after ventilation. Mice were subjected to mechanical ventilation $(30 \mathrm{ml} / \mathrm{kg})$ for $4 \mathrm{~h}$. Salidroside was intraperitoneally administered $1 \mathrm{~h}$ before the onset of ventilation. The left lower lung was fixed, embedded in paraffin, and used for histological evaluation by hematoxylin and eosin staining. (A) Control group; (B) ventilation group; (C) Salidroside group; (D) Ventilation + Salidroside group. Original magnification, $\times 200$. Scale bars correspond to $100 \mu \mathrm{m}$. The severity of lung injury(E) was scored. Data are expressed as the mean \pm SEM ( $\mathrm{n}=7$ ). ${ }^{* *} \mathrm{p}<0.01$ vs control group; \#\#p<0.01 vs ventilation group.
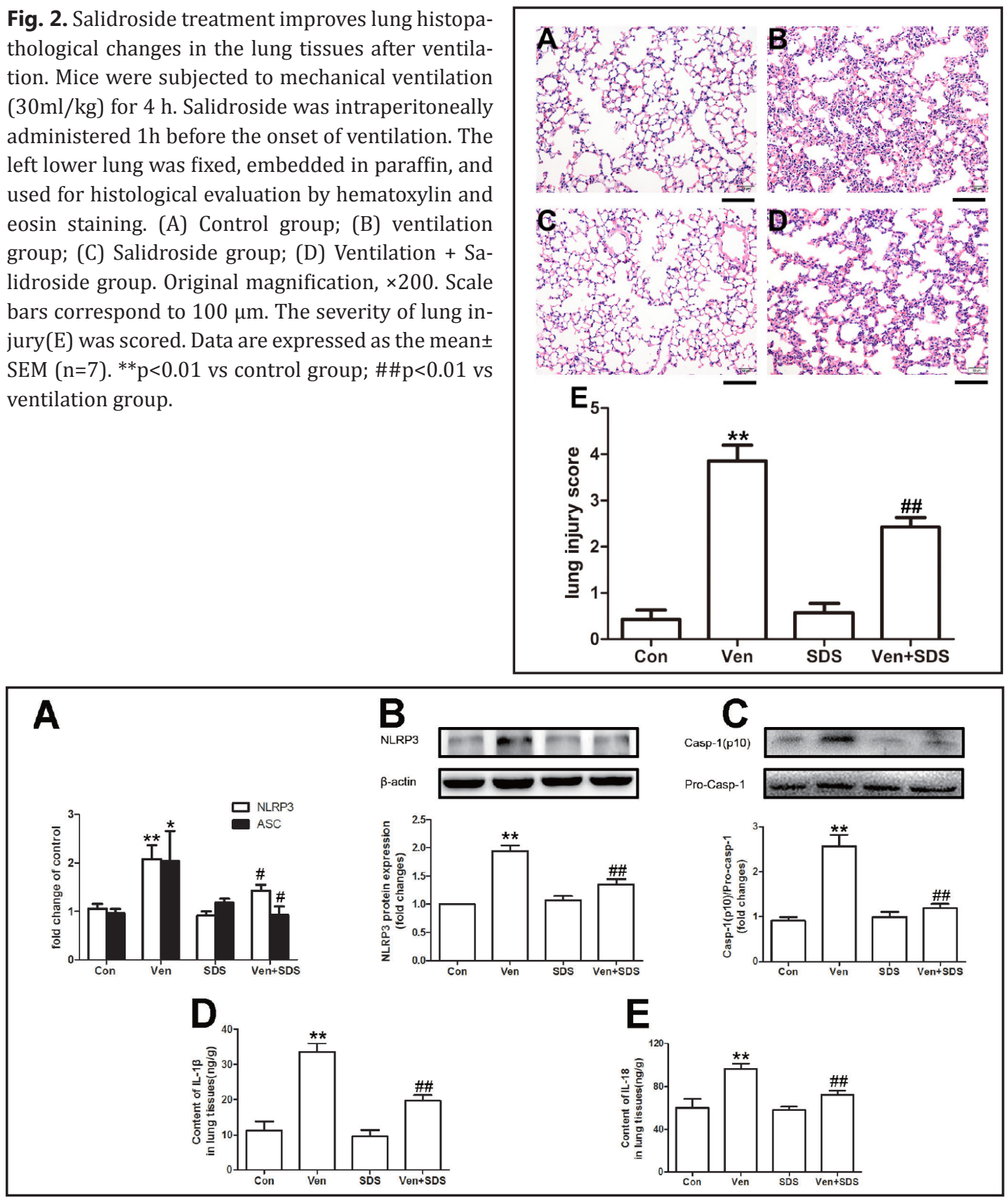

Fig. 3. Salidroside inhibits ventilation-induced activation of NLRP3 inflammasome in vivo. Mice were subjected to mechanical ventilation $(30 \mathrm{ml} / \mathrm{kg}$ ) for $4 \mathrm{~h}$. Salidroside was intraperitoneally administered $1 \mathrm{~h}$ before the onset of ventilation. NLRP3 and ASC mRNA levels(A), IL-1B(D) and IL-18(E) content in lung tissues were determined by quantitative real-time PCR and ELISA, respectively. Protein expression of NLRP3 and pro-Caspase- 1 and Caspase-1 p10 fragment in lung homogenates were determined by Western blot analysis. Relative densitometry of NLRP3/ $\beta$-actin(B) and caspase- 1 p10/pro-Caspase-1(C) ratio was shown in bar graphs. The representative protein bands were presented on the top of the bar graphs. Data are expressed as means \pm SEM $(n=7)$. ${ }^{*} \mathrm{P}<0.05,{ }^{* *} \mathrm{P}<0.01$ vs control group; $\# \mathrm{P}<0.05$, \#\# $\mathrm{P}<0.01$ vs ventilation group.

Salidroside inhibits mechanical stretch-induced activation of NLRP3 inflammasome in MLVECS

Lung microvascular overdistension during mechanical ventilation at high tidal volume can cause increased lung vascular leak, which is a result of stretch-induced endothelial KARGER 

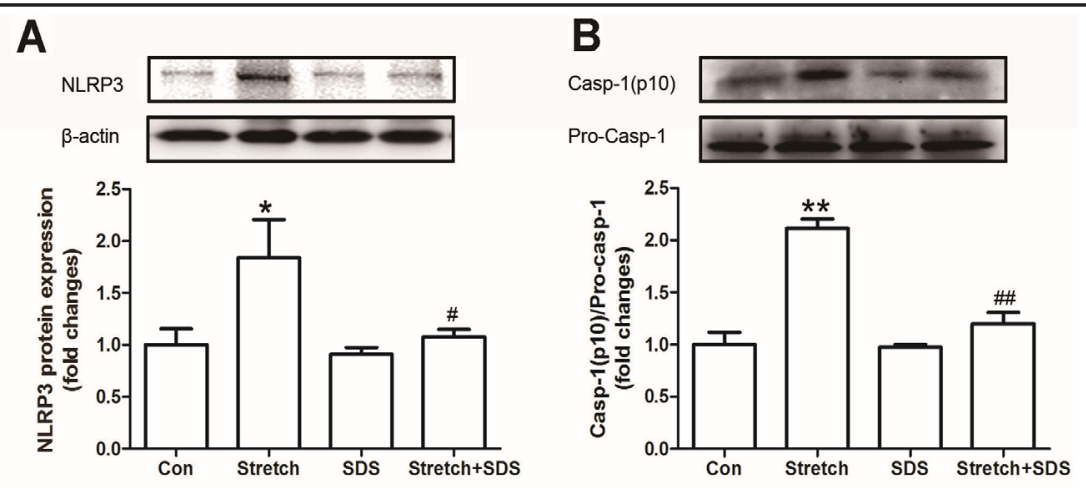

Fig. 4. Salidroside inhibits ventilation-induced activation of NLRP3 inflammasome in vitro. Primary cultured MLVECs were pretreated with salidroside and then stimulated with cyclic stretch. Four hours after cyclic stretch, cells were collected for determining protein levels of NLRP3 and caspase-1. Relative densitometry of NLRP3/ $\beta$-actin(A) and caspase-1 p10/pro-Caspase-1(B) ratio was shown in bar graphs. The representative protein bands were presented on the top of the bar graphs. Data are expressed as means \pm SEM $(n=4)$. * $\mathrm{P}<0.05$, ** $\mathrm{P}<0.01$ vs control group; \# $\mathrm{P}<0.05$, \#\# $\mathrm{P}<0.01$ vs stretch group.

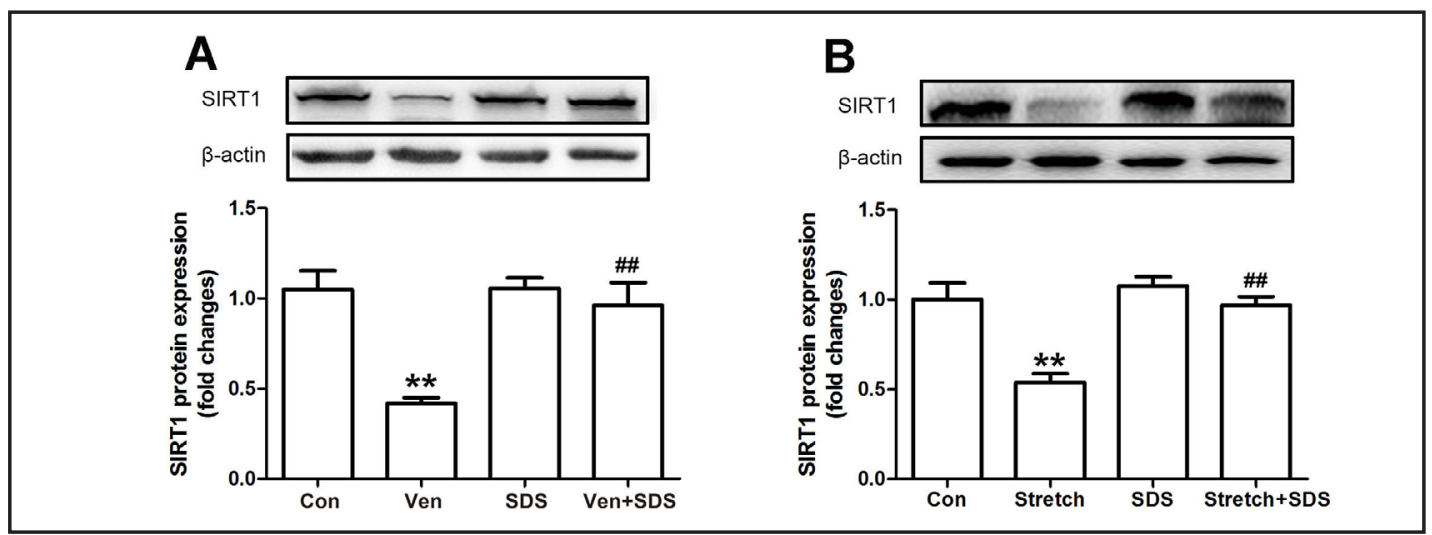

Fig. 5. Salidroside up-regulated SIRT1 in vivo and in vitro. Mice were subjected to mechanical ventilation $(30 \mathrm{ml} / \mathrm{kg})$ for $4 \mathrm{~h}$. Salidroside was intraperitoneally administered $1 \mathrm{~h}$ before the onset of ventilation. Protein expression of SIRT1 in lung homogenates was determined by Western blot analysis. Relative densitometry of SIRT1/ $\beta$-actin(A) was shown in bar graphs. The representative protein bands were presented on the top of the bar graphs(n=7). Primary cultured MLVECs were pretreated with salidroside and then stimulated with cyclic stretch. Four hours after cyclic stretch, cells were collected for determining protein levels of SIRT1. Relative densitometry of SIRT1/ $\beta$-actin(B) was shown in bar graphs. The representative protein bands were presented on the top of the bar graphs. Data are expressed as means \pm SEM $(n=4)$. ${ }^{* *} \mathrm{P}<0.01$ vs control group; \#\# $\mathrm{P}<0.01$ vs ventilation group or stretch group.

barrier dysfunction [18]. Therefore, we then examined the effects of mechanical stretch and SDS on NLRP3 inflammasome activation in primary cultured mouse lung microvascular endothelial cells (MLVECs) in vitro. As expected, exposure to cyclic stretch for $4 \mathrm{~h}$ resulted in profound increases in NLRP3 protein expression and cleaved caspase-1 p10 level in primary cultured MLVECs (Fig. 4), indicating that cyclic stretch alone had the ability to induce NLRP3 activation in MLVECs. Salidroside treatment significantly inhibited the cyclic stretch-induced activation of NLRP3 inflammasome in MLVECs (Fig. 4).

Salidroside up-regulates SIRT1 in vivo and in vitro

Gao et al. have suggested the involvement of SIRT1 in the inflammatory response of vascular cells [19].We then explored whether SIRT1 was involved in the inhibitory effect of 
A
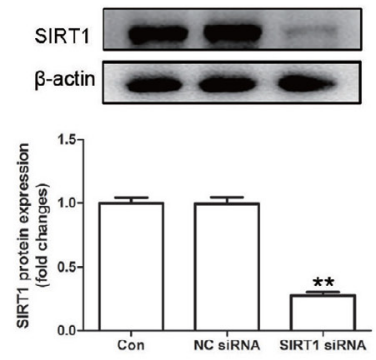

B

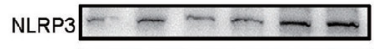

$\beta$-actin $-\infty-\infty$

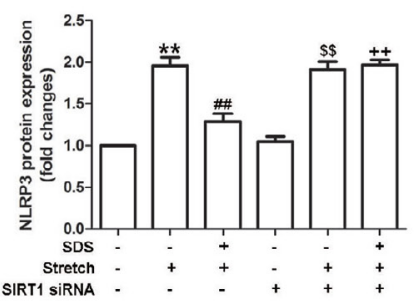

\section{C}

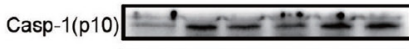

Pro-Casp-1 ---

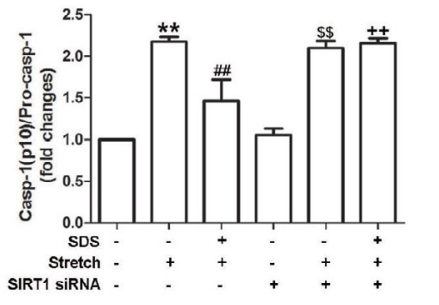

Fig. 6. Salidroside inhibits Cyclic Stretch-induced the activation of NLRP3 inflammasome via SIRT1. Primary cultured MLVECs were transfected with negative control siRNA or SIRT1 siRNA for 24h. The expression of SIRT1(A) was examined by western blot. Twenty-four hours later, the culture medium was changed, and the cells were treated with cyclic stretch. Four hours after cyclic stretch, cells were collected for determining protein levels of NLRP3 and caspase-1. Relative densitometry of NLRP3/ $\beta$-actin(B) and caspase-1 p10/pro-Caspase-1(C) ratio was shown in bar graphs. The representative protein bands were presented on the top of the bar graphs. Data are expressed as mean \pm SEM $(n=4)$. ${ }^{* *} \mathrm{P}<0.01$ vs control group; \#\# $\mathrm{P}<0.01$ vs stretch group; $\$$ P $<0.01$ vs SIRT1 siRNA treatment; ++ $\mathrm{P}<0.01$ vs cyclic stretch + salidroside treatment.

salidroside on NLRP3 inflammasome activation. By western blot analysis, we showed that ventilation obviously down-regulated the expression of SIRT1 in lung tissues, whereas the ventilation-mediated down-regulation was reversed by salidroside (Fig. 5A).

In addition, in primary cultured MLVECs we showed that cyclic stretch led to a significant decrease in SIRT1 expression, which was reversed by salidroside treatment (Fig. 5B).

Salidroside inhibits Cyclic Stretch-induced the activation of NLRP3 inflammasome via SIRT1

We then investigated whether SIRT1 played a key role in the inhibitory effect of salidroside on NLRP3 inflammasome activation. Accordingly, SIRT1 siRNA was applied in our study. MLVECs were transfected with SIRT1 siRNA, and resulted in an approximately $70 \%$ decrease in SIRT1 expression as compared with those transfected with control siRNA (Fig. 6A). The transfected MLVECs were treated with Salidroside $(50 \mu \mathrm{M})$, and subsequently exposed to Cyclic Stretch. We found that the inhibitory effect of Salidroside on cyclic stretch-induced increases in NLRP3 protein expression in MLVECs was abolished by SIRT1 knockdown, indicating that regulation of NLRP3 expression by Salidroside depends on SIRT1-dependent pathway (Fig. 6B and 6C). These results indicate that the modulatory effect of salidroside on NLRP3 inflammasome activation in cyclic stretch-stimulated MLVECs is mediated by SIRT1.

\section{Discussion}

ALI is a devastating clinical syndrome with the characteristics of acute lung inflammation and vascular barrier disruption and is responsible for the high mortality rate observed in ARDS patients. Mechanical ventilation, especially with HTV, can worsen or even cause lung injury [1-3]. Direct contribution of NLRP3 inflammasome in the lung vascular barrier dysfunction has been shown in the animal models of ALI/VILI and in pulmonary endothelial cell monolayers exposed to pathological cyclic stretch [5]. In turn, the activation of NLRP3 inflammasome in the animal models of ALI/VILI caused lung endothelial hyperpermeability and reduced indices of lung injury in the animal models of ALI/VILI. In the present study, we demonstrated that SDS treatment significantly ameliorated the symptoms of VILI as measured by HE staining, cell count and protein content levels in BAL fluid, W/D and Evans 
blue dye leakage into the lung tissue. Herein, our observations implied that SDS inhibited the activation of NLRP3 inflammasome, as measured by decreased NLRP3 and ASC expression and suppressed caspased- 1 and IL-1 $\beta$ maturation both in vivo and in vitro study. Furthermore, our data also showed that SDS exerted an inhibitory effect partly through up-regulation of SIRT1 expression.

To the best of our knowledge, it is the first time to give evidence of the effect of SDS in ventilation-induced lung injury mice. The effect of salidroside on various models of lung injury has been reported. In a LPS-induced acute lung injury(ALI) model, salidroside exerted anti-inflammatory effect via down-regulating the activation of JAK2-STAT3 pathway and suppressing STAT3 transfer into the nucleus in part [20]. In septic mice, Liu et al. show that salidroside administration prolongs the survival, alleviates the pathologic lung changes and preserves adaptive immunity [21]. Likewise, in an ovalbumin (OVA)-induced asthma model, after treatment of salidroside, the airway hyper-reactivity virtually eliminates, the eosinophil percent markedly reduces, the level of IL-4 reduces and INF- $\gamma$ obviously raises, and this is related to the regulation of Th1/Th2 balance [22]. In these models, salidroside shows its anti-inflammation and antioxidation effects. Likewise, in the present study, we showed the protective effect of SDS in ventilation induced lung injury, and this effect may be mediated via inhibiting the activation of NLRP3 both in the animal models and in microvessel endothelial cells exposed to pathological cyclic stretch.

As we known, NLRP3 inflammasome and its effector cytokines are implicated in both acute and chronic pulmonary disorders $[4,23]$. Previous studies have shown that pulmonary fibrosis is mediated by the NLRP3 inflammasome [24]. It is of interest that salidroside has been demonstrated to have protective effects against pulmonary fibrosis. Tang et al. demonstrate that in bleomycin-induced pulmonary fibrosis salidroside can inhibit IкB $\alpha$ phosphorylation and nuclear factor kappa $\mathrm{B}(\mathrm{NF}-\kappa \mathrm{B})$ via activating Nrf2-antioxidant signaling [25]. The recombinant TGF- $\beta 1$-induced EMT-like changes can be reversed and the expression of TGF- $\beta 1$ and the phosphorylation of its downstream targets, Smad-2/-3, were weakened by salidroside. In recent years, studies have shown that mechanical ventilation can cause pulmonary fibrosis [26], then the mechanism of SDS in inhibiting NLRP3 inflammasome can reduce ventilation induced pulmonary fibrosis, it is worth further research.

SIRT1 is known to play a pivotal role in preventing cells from injury induced by various stress during ischemia reperfusion or inflammation and is involved in the inhibition of NLRP3 inflammasome [9, 27]. In LPS and ATP-induced activation of NLRP3 inflammasome, SIRT1 is demonstrated to have an inhibitory effect by decreasing CD40 and CD40L levels [28]. And Nicotinic acid can regulate the activation of NLRP3 inflammasome partly through SIRT1 and ROS [29]. Misawa et al. also report that SIRT1 activator treatment significantly suppressed the maturation of caspase-1 in mouse macrophages, which means SIRT1 may be linked to the regulation of the NLRP3 inflammasome [30]. In the present study, we showed the inhibitory effect of salidroside on the NLRP3 inflammasome and the subsequent cytokines, and these effects could be abolished by SIRT1 knockdown, which mean the NLRP3 inflammasome could be regulated by SIRT1. Although we have gained promising data concerning the protective effect of SDS in lung injury and the regulation mechanism, several limitations of the present study need to be addressed such as the explicit molecular mechanism underlying the regulation and how SIRT1 regulated the NLRP3 inflammasome.

As is known, SIRT1 is an NAD+-dependent deacetylase [31]. In macrophage, the NLRP3 inflammasome can be regulated by the mitochondrial-enriched sirtuin deacetylase SIRT3 [32]. Hwang et al. demonstrate HDAC6 regulates the activation of NLRP3 inflammasome by its direct interaction to ubiquitinate NLRP3 [33]. Whether SIRT1 regulates NLRP3 inflammasome via ubiquitination need to be clarified in the future.

Despite the rapid improvements in intensive care medicine, VILI is still a life-threatening and increasing frequent disease [1,34]. Numerous scholars have elucidated the pathogenesis of VILI, however little progress has been made in its pharmacologic management. Clinicians are still in therapeutic dilemma to accomplish effective strategies for VILI. In the present study, we demonstrated that the activation of NLRP3 inflammasome might act as a trigger 
for the development of VILI. Moreover, salidroside was exerted protective effects against ventilation induced lung injury via inhibiting the NLRP3 inflammasome. These findings raise the possibility that salidroside may represent a useful candidate for preventing VILI.

In conclusion, we explored the anti-inflammatory effects of SDS administration in a mouse model of VILI and pathological cyclic stretch in microvessel endothelial cells, and found that the inflammatory process was significantly attenuated by SDS treatment. The mechanisms behind the inhibitory effect of SDS on the activation of NLRP3 inflammasome may be related to regulation of SIRT1. The findings obtained in this study provide a significant insight of the protective effect of salidroside and suggest a new area of the anti-inflammatory effects of SDS for treating VILI.

\section{Disclosure Statement}

The authors of this article have no conflict of interest. This work was supported by grants from the National Natural Science Foundation of China to Dr. Jiang (No. 81571929, No. 81272144), Dr. Zhu (No. 31671213, No. 31271270), Dr. Mao (No. 81372100) and Dr. Liu (No.81672266, No.31371164).

\section{References}

- Goligher EC, Doufle G, Fan E:Update in Mechanical Ventilation, Sedation, and Outcomes 2014. Am J Respir Crit Care Med 2015;191:1367-1373.

-2 Choi WI, Quinn DA, Park KM, Moufarrej RK, Jafari B, Syrkina O, Bonventre JV, Hales CA: Systemic microvascular leak in an in vivo rat model of ventilator-induced lung injury. Am J Respir Crit Care Med 2003;167:1627-1632.

-3 Matthay MA,Zimmerman GA, Esmon C, Bhattacharya J, Coller B, Doerschuk CM, Floros J, Gimbrone MA Jr, Hoffman E, Hubmayr RD, Leppert M, Matalon S, Munford R, Parsons P, Slutsky AS, Tracey KJ, Ward P, Gail DB, Harabin AL: Future research directions in acute lung injury: summary of a National Heart, Lung, and Blood Institute working group. Am J Respir Crit Care Med 2003;167:1027-1035.

-4 Abderrazak A, Syrovets T, Couchie D, El Hadri K, Friguet B, Simmet T, Rouis M: NLRP3 inflammasome: from a danger signal sensor to a regulatory node of oxidative stress and inflammatory diseases. Redox Biol 2015;4:296-307.

-5 Kuipers MT, Aslami H, Janczy JR, van der Sluijs KF, Vlaar AP, Wolthuis EK, Choi G, Roelofs JJ, Flavell RA, Sutterwala FS, Bresser P, Leemans JC, van der Poll T, Schultz MJ, Wieland CW: Ventilator-induced lung injury is mediated by the NLRP3 inflammasome. Anesthesiology 2012;116:1104-1115.

6 Latz E, Xiao TS, Stutz A: Activation and regulation of the inflammasomes. Nat Rev Immunol 2013;13:397-411.

7 Yu G, Zeng X, Wang H, Hou Q, Tan C, Xu Q, Wang H: 14,15-epoxyeicosatrienoic Acid suppresses cigarette smoke extract-induced apoptosis in lung epithelial cells by inhibiting endoplasmic reticulum stress. Cell Physiol Biochem 2015;36:474-486.

-8 Wang WR, Liu EQ Zhang JY, Li YX, Yang XF, He YH, Zhang W, Jing T, Lin R: Activation of PPAR alpha by fenofibrate inhibits apoptosis in vascular adventitial fibroblasts partly through SIRT1-mediated deacetylation of Fox01. Exp Cell Res 2015;338:54-63.

-9 Fu Y, Wang Y, Du L, Xu C, Cao J, Fan T, Liu J, Su X, Fan S, Liu Q, Fan F: Resveratrol inhibits ionising irradiationinduced inflammation in MSCs by activating SIRT1 and limiting NLRP-3 inflammasome activation. Int J Mol Sci 2013;14:14105-14118.

10 Li Y, Wang P, Yang X, Wang W, Zhang J, He Y, Zhang W, Jing T, Wang B, Lin R: SIRT1 inhibits inflammatory response partly through regulation of NLRP3 inflammasome in vascular endothelial cells. Mol Immunol 2016;77:148-156.

11 Qian EW, Ge DT, Kong SK: Salidroside protects human erythrocytes against hydrogen peroxide-induced apoptosis. J Nat Prod 2012;75:531-537.

12 Song B, Huang G, Xiong Y, Liu J, Xu L, Wang Z, Li G, Lu J, Guan S: Inhibitory effectsof salidroside on nitric oxide and prostaglandin E2 production inlipopolysaccharide-stimulated RAW 264.7 macrophages. J Med Food 2013;16:997-1003. 


\section{Cellular Physiology Cell Physiol Biochem 2017;42:34-43 \begin{tabular}{l|l} 
DOI: 10.1159/000477112 & $\begin{array}{l}\text { O 2017 The Author(s). Published by S. Karger AG, Basel } \\
\text { www.karger.com/cpb }\end{array}$ \\
\hline
\end{tabular}}

Wang et al.: SDS Attenuates VILI

13 Zhang Y, Yao Y, Wang H, Guo Y, Zhang H, Chen L: Effects of salidroside on glioma formation and growth inhibition together with improvement of tumor microenvironment. Chin J Cancer Res 2013;25:520-526.

-14 Zhu Y, Shi YP, Wu D, Ji YJ, Wang X, Chen HL, Wu SS, Huang DJ, Jiang W: Salidroside protects against hydrogen peroxide-induced injury in cardiac H9c2 cells via PI3K-Akt dependent pathway. DNA Cell Biol 2011;30:809-819.

15 Guan S, Xiong Y, Song B, Song Y, Wang D, Chu X, Chen N, Huo M, Deng X, Lu J: Protective effects of salidroside from Rhodiola rosea on LPS-induced acute lung injury in mice. Immunopharmacol Immunotoxicol 2012;34:667-672.

-16 Zhang Z, Ding L, Wu L, Xu L, Zheng L, Huang X: Salidroside alleviates paraquat-induced rat acute lung injury by repressing TGF- $\beta 1$ expression. Int J Clin Exp Pathol 2014;7:8841-8847.

17 Dong WW, Liu YJ, Lv Z, Mao YF, Wang YW, Zhu XY, Jiang L:Lung endothelial barrier protection by resveratrol involves inhibition of HMGB1 release and HMGB1-induced mitochondrial oxidative damage via an Nrf2dependent mechanism. Free Radic Biol Med 2015;88:404-416.

- 18 Birukova AA, Fu P, Xing J, Yakubov B, Cokic I, Birukov KG:Mechanotransduction by GEF-H1 as a novel mechanism of ventilator-induced vascular endothelial permeability. Am J Physiol Lung Cell Mol Physiol 2010;298:837-848.

19 Gao P, Xu TT, Lu J, Li L, Xu J, Hao DL, Chen HZ, Liu DP: Overexpression of SIRT1 in vascular smooth muscle cells attenuates angiotensin II-induced vascular remodeling and hypertension in mice. J Mol Med (Berl) 2014;92:347-357.

20 Qi Z, Qi S, Ling L, Lv J, Feng Z: Salidroside attenuates inflammatory response via suppressing JAK2-STAT3 pathway activation and preventing STAT3 transfer into nucleus. Int Immunopharmacol 2016;35:265-271.

21 Liu S, Yu X, Hu B, Zou Y, Li J, Bo L, Deng X: Salidroside rescued mice from experimental sepsis through antiinflammatory and anti-apoptosis effects. J Surg Res 2015;195:277-283.

22 Wang J, Jin RG, Xiao L, Wang QJ, Yan TH: Anti-asthma effects of synthetic salidroside through regulation of Th1/Th2 balance. Chin J Nat Med 2014;12:500-504.

23 Huang Z, Zhuang X, Xie C, Hu X, Dong X, Guo Y, Li S, Liao X: Exogenous Hydrogen Sulfide Attenuates High Glucose-Induced Cardiotoxicity by Inhibiting NLRP3 Inflammasome Activation by Suppressing TLR4/NFкB Pathway in H9c2 Cells. Cell Physiol Biochem 2016;40:1578-1590.

24 Liu W, Zhang X, Zhao M, Zhang X, Chi J, Liu Y, Lin F, Fu Y, Ma D, Yin X: Activation in M1 but not M2 Macrophages Contributes to Cardiac Remodeling after Myocardial Infarction in Rats: a Critical Role of the Calcium Sensing Receptor/NRLP3 Inflammasome. Cell Physiol Biochem 2015;35:2483-2500.

25 Tang H, Gao L, Mao J, He H, Liu J, Cai X, Lin H, Wu T: Salidroside protects against bleomycin-induced pulmonary fibrosis: activation of Nrf2-antioxidant signaling, and inhibition of NF-kappaB and TGF-beta1/ Smad-2/-3 pathways. Cell Stress Chaperones 2016;21:239-249.

-26 Burnham EL, Janssen WJ, Riches DW, Moss M, Downey GP: The fibroproliferative response in acute respiratory distress syndrome: mechanisms and clinical significance. Eur Respir J 2014;43:276-285.

-27 Ruan Y, Dong C, Patel J, Duan C, Wang X, Wu X, Cao Y, Pu L, Lu D, Shen T, Li J: SIRT1 suppresses doxorubicininduced cardiotoxicity by regulating the oxidative stress and p38MAPK pathways. Cell Physiol Biochem 2015;35:1116-1124.

28 Li Y, Yang X, He Y, Wang W, Zhang J, Zhang W, Jing T, Wang B, Lin R: Negative regulation of NLRP3 inflammasome by SIRT1 in vascular endothelial cells. Immunobiology2016;pii:S0171-2985.

29 Li Y, Yang G, Yang X, Wang W, Zhang J, He Y, Zhang W, Jing T, Lin R: Nicotinic acid inhibits NLRP3 inflammasome activation via SIRT1 in vascular endothelial cells. Int Immunopharmacol 2016;40:211-218.

-30 Misawa T, Saitoh T, Kozaki T, Park S, Takahama M, Akira S: Resveratrol inhibits the acetylated $\alpha$-tubulinmediated assembly of the NLRP3-inflammasome. Int Immunol 2015;27:425-434.

-31 Yamamoto H, Schoonjans K, Auwerx J:Sirtuin functions in health and disease. Mol Endocrinol 2007;21:1745-1755.

- 32 Traba J, Kwarteng-Siaw M, Okoli TC, Li J, Huffstutler RD, Bray A, Waclawiw MA, Han K, Pelletier M, Sauve AA, Siegel RM, Sack MN: Fasting and refeeding differentially regulate NLRP3 inflammasome activation in human subjects. J Clin Invest 2015;125:4592-4600.

33 Hwang I, Lee E, Jeon SA, Yu JW: Histone deacetylase 6 negatively regulates NLRP3 inflammasome activation. Biochem Biophys Res Commun2015;467:973-978.

-34 Ventilation with lower tidal volumes as compared with traditional tidal volumes for acute lung injury and the acute respiratory distress syndrome. The Acute Respiratory Distress Syndrome Network. N Engl J Med 2000;342:1301-1308. 